\title{
VARIATIONAL PRINCIPLES WITH LINEAR GROWTH
}

\author{
By \\ Robert Hardt \\ and \\ David Kinderlehrer
}

IMA Preprint Series \# 540

June 1989 
"Partial Differential Equations and the Calculus

of Variations: Essays in Honor of Ennio De Giorgi"

Progress in Nonlinear Differential Equations and their Applications

Birkhaüser Boston Inc., 1989.

\section{VARIATIONAL PRINCIPLES WITH LINEAR GROWTH}

ROBERT HARDT

David Kinderlehrer

Dedicated to Ennio De Giorgi on his sixtieth birthday

Variational principles which exhibit only linear growth arise in several contexts. As a paradigm for the questions which these principles suggest, we take up here the study of the problem

$$
\inf _{v \in \mathcal{A}}\left\{\int_{\Omega} \phi(\nabla v) d x-\int_{\Omega} f v d x\right\}
$$

Here $\phi: \mathbf{R}^{n} \rightarrow \mathbf{R}$ is a non-negative convex sufficiently differentiable function satisfying

$$
\phi(0)=0, \phi_{p}(0)=0, \text { and }|p|-\lambda \leq \phi \leq|p|
$$

for some $\lambda>0$, subject to

$$
\lim _{t \rightarrow \infty} \phi(t p) / t|p|=1
$$

The competing $v$ belong to a suitable class $\mathcal{A}$ of functions from $\Omega$ to $\mathbf{R}$ where $\Omega$ is a bounded domain with sufficiently smooth boundary and $f$ is given. 
Our discussion applies in particular to the integrand

$$
\phi(p)= \begin{cases}\frac{1}{2}|p|^{2} & \text { for }|p| \leq 1 \\ |p|-\frac{1}{2} & \text { for }|p| \geq 1\end{cases}
$$

which arises in the study of anti-planar shear in elastic/plastic deformation, $\left[H K_{1}\right]$, $[\mathrm{KT}]$, [ST].

A solution to (0.1) may be found direct methods or by resorting to a generalized principle of complementary energy. The solution found by direct methods needs not be unique and, pertinent here, its gradient may be only a measure. The solution of the dual problem of complementary energy is often unique and determines, in case of (0.4), the region associated to elastic and plastic behavior.

Our concerns here will include properties of the generalized stress, the solution of the dual problem, which lead to information about the direct problem and its consequent smoothness.

These issues were brought to our attention by the work of Anzellotti and Giaquinta $\left(\left[A G_{1}\right],\left[A G_{2}\right]\right)$ who treated the direct problem, and Strang and Temam [ST] who investigated both the direct problem and the dual problem. As we have indicated, it was partly our desire to achieve a greater unity between these approaches which led us to take up this argument. Our method is transparent and may be summarized in a few words: We have attempted to apply what we have learned from our study of the work of Ennio De Giorgi. It is with special pleasure that we dedicate this paper to him.

Central to our work is the study of the $(n-1)$ density

$$
\Theta(a)=\lim _{r \rightarrow 0} r^{1-n} \int_{B_{r}(a)}|D u|
$$

for a local solution $u$ of $(0.1)$. In $\S 1$ we show that this density exists and is upper semi-continuous in $\Omega$. In $\S 2$, we show that the local solution $u$ is continuous at a point $a \in \Omega$ if and only if its $(n-1)$ density vanishes at that point. In $\S 3$ the behavior near points of positive density is investigated, and it is shown how the solution is, in a very strong sense, essentially two-sided continuous, with the exception of a set of $n-1$ dimensional measure zero. The curvature of the set of discontinuities, or slip set, is then determined by the inhomogeneous term $f$. 
Some of the present work was announced in $\left[\mathrm{HK}_{2}\right]$, the latter containing some discussion of open questions. We wish to point out some more recent work in this general area, $\left[A G_{3}\right]$, and dynamical problems, [RS], [AL].

Implicit in our introduction of the $(n-1)$ density $\Theta(a)$ and our subsequent discussion is the recognition that we shall be obliged to widen our class of admissible funtions to $B V(\Omega)$. To further motivate this, we briefly review the dual problem associated to $(0.1)$.

Assuming that $u_{0} \in H^{1,1}(\Omega)$ and $f \in L^{\infty}(\Omega)$, set

$$
\mathcal{A}=\left\{v \in H^{1,1}(\Omega): v=u_{0} \text { on } \partial \Omega\right\} .
$$

Defining

$$
I(v)=\int_{\Omega} \phi(\nabla v) d x-\int_{\Omega} f v d x, v \in \mathcal{A},
$$

we may extend our functional to $H^{1,1}(\Omega)$ by [ET]

$$
I(v)=\int_{\Omega} \phi(\nabla v) d x+I_{\mathcal{A}}(v)-F(v)
$$

where

$$
I_{A}(v)= \begin{cases}0 & v \in \mathcal{A} \\ \infty & v \notin \mathcal{A}\end{cases}
$$

is the indicator function of $A$ and

$$
F(v)=\int_{\Omega} f v d x .
$$

Our original variational principle (0.1) now becomes a problem

(P) To find the solution or characteristic extremals of

$$
i=\inf _{H^{1,1}(\Omega)}\left\{\int_{\Omega} \phi(\nabla v) d x+I_{\mathcal{A}}(v)-F(v)\right\} .
$$

We may easily calculate the dual functional $I^{*}(\tau)$ for a vectorfield $\tau$. Indeed,

$$
I^{*}(\tau)= \begin{cases}\int_{\Omega} \phi^{*}(-\tau) d x+\int_{\Omega} \tau \cdot \nabla u_{0} d x+\int_{\Omega} f u_{0} d x & \text { if } \operatorname{div} \tau=f \\ +\infty & \text { otherwise }\end{cases}
$$


Several properites of the dual functional $\phi^{*}$ are worthy of note. First observe that $(0.2)$ and the convexity of $\phi$ imply that

$$
\left|\phi_{p}(p)\right| \leq 1 \text { whenever } 0 \neq p \in \mathbf{R}^{n} .
$$

Now

$$
\phi^{*}(q)= \begin{cases}p \cdot \phi_{p}(p)-\phi(p) & \text { whenever } q=\phi_{p}(p) \text { and }|q| \leq 1 \\ +\infty & \text { whenever }|q|>1 .\end{cases}
$$

Again using (0.2), we obtain the estimate, useful later on,

$$
0 \leq \phi^{*}(q) \leq \lambda \text { if }|q| \leq 1 .
$$

Since (0.6) implies that

$$
\int_{\Omega} \phi^{*}(-\tau) d x=+\infty \text { if }\|\tau\|_{L^{\infty}(\Omega)}>1,
$$

we may further restrict the competitors which will occur in the dual principle. We arrive at the problem

$\left(P^{*}\right)$ To find or characterize extremals of

$$
i^{*}=\inf _{\operatorname{div} \tau=f,|\tau| \leq 1}\left\{\int_{\Omega} \phi^{*}(-\tau) d x+\int_{\Omega} \tau \cdot \nabla u_{0} d x+\int_{\Omega} f u_{0} d x\right\} .
$$

The theory of convex duality ([ET] or $\left.\left[\mathrm{B}_{3}\right], \mathrm{p} .8\right)$ assures us that whenever

$$
\mathcal{A}^{*}=\left\{\tau \in L^{\infty}(\Omega)^{n}:|\tau| \leq 1 \text { and } \operatorname{div} \tau=f\right\}
$$

is not empty, there exists a $\sigma \in \mathcal{A}^{*}$ such that

$$
i=i^{*}=\int_{\Omega} \phi^{*}(-\sigma) d x+\int_{\Omega} \sigma \cdot \nabla u_{0} d x+\int_{\Omega} f u_{0} d x .
$$

Indeed, $\left(P^{*}\right)$ is a variational inequality [S].

In the special case $(0.4)$ the dual function is

$$
\phi^{*}(\sigma)= \begin{cases}\frac{1}{2}|\sigma|^{2} & \text { for }|\sigma| \leq 1 \\ \infty & \text { for }|\sigma|>1\end{cases}
$$


and the dual functional is

$$
I^{*}(\tau)=\frac{1}{2} \int_{\Omega}|\tau|^{2} d x+\int_{\Omega} \tau \cdot \nabla u_{0} d x+\int_{\Omega} f u_{0} d x \text { whenever } \tau \in \mathcal{A}^{*}
$$

However, there need not be $u \in \mathcal{A}$ whose stress achieves this infimum, and thus we are led to search for $u$ in the wider class $B V(\Omega)$ of functions whose gradients are measures.

A second motivation is the absence of a suitable Lagrangian functional which enables us to pair vectorfields $\tau$ and $v$ in $H^{1,1}(\Omega)$. Determining the validity of the Lagrangian in an extended sense is one of the subjects treated by Kohn and Temam [KT]. Here we limit ourselves to the relationship between the solution of $(0.1)$ or $(0.7)$ and its own stress vector.

The important question of when $\mathcal{A}^{*}$ is not empty or when $I(v)$ is bounded below on $\mathcal{A}$ may be resolved in several ways, c.f.[ $\left.A G_{1}\right],[\mathrm{KT}]$, $\left[H K_{1}\right]$. For example, given $\Omega$ there is a constant $C_{\Omega}$ such that

$$
\|\zeta\|_{L^{1}(\Omega)} \leq C_{\Omega}\|\zeta\|_{B V(\Omega)} \text { for } \zeta \in B V_{0}(\Omega)
$$

Thus $I(v)$ is bounded below whenever

$$
\|f\|_{L^{\infty}(\Omega)}<C_{\Omega}^{-1} .
$$

Methods of limit analysis offer an alternative view, $\left[T_{1}\right]$.

In our motivation we have discussed a variational principle with Dirichlet boundary conditions. Neumann conditions may be considered as well.

Let $\Omega \subset \mathbf{R}^{n}$ be a bounded domain with Lipschitz boundary. Given $v \in B V(\Omega)$, we decompose its gradient measure $D v$ into its absolutely continuous and singular parts with respect to Lebesgue measure:

$$
D v=\nabla v d x+D^{s} v
$$

We define

$$
I(v)=\int_{\Omega} \phi(D v)=\int_{\Omega} \phi(\nabla v) d x+\int_{\Omega}\left|D^{s} v\right|
$$

This definition is motivated primarily by the fact that there exist $v_{\delta} \in C^{\infty}(\bar{\Omega}), 0<\delta<1$, such that $\left(v_{\delta}\right)$ is contained in a bounded set in $B V(\Omega)$ and

$$
v_{\delta} \rightarrow v \text { in } L^{\frac{n}{n-1}} \cap L^{1}(\partial \Omega) \text { and } I\left(v_{\delta}\right)=\int_{\Omega} \phi\left(\nabla v_{\delta}\right) d x \rightarrow I(v)
$$


as $\delta \rightarrow 0$, c.f.[AG $]$,[H $\left[H K_{1}\right.$,Th.A.2]. The stress associated to $v$ is defined by

$$
\sigma=\sigma(v)= \begin{cases}\phi_{p}(\nabla v) & \text { in } \Omega_{a} \\ D^{s} v /\left|D^{s} v\right| & \text { in } \Omega_{s}\end{cases}
$$

where $D^{s} v /\left|D^{s} v\right|$ is the Radon-Nikodym derivative of $D^{s} v$ with respect to its total variation measure $\left|D_{s} v\right|$ and $\Omega=\Omega_{a} \cup \Omega_{s}$ is the decomposition of $\Omega$ with respect the mutually singular measures $d x$ and $\left|D^{s} v\right|$.

We have already noticed that since $\phi$ is convex and (0.2) holds,

$$
\left|\phi_{p}(p)\right| \leq 1 \text { and } \phi(p) \leq \phi_{p}(p) \cdot p
$$

It follows that

$$
|\sigma(v)| \leq 1 \text { and }
$$

$$
\phi(D v)=\phi(\nabla v)+\left|D^{s} v\right| \leq \phi_{p}(\nabla v) \cdot \nabla v+\left|D^{s} v\right|=\sigma(v) \cdot D v
$$

1. Local solutions and variational formulas. We say that a function $u \in B V(\Omega)$ is a local solution in $\Omega$ provided that

$$
\int_{\Omega} \phi(D u)-\int_{\Omega} f u d x \leq \int_{\Omega} \phi(D(u+\zeta))-\int_{\Omega} f(u+\zeta) d x \text { for } \zeta \in B V_{0}(\Omega) .
$$

For any $\zeta \in B V_{0}(\Omega)$ satisfiyng

$$
D^{s} \zeta \ll\left|D^{s} u\right|,
$$

we have the first variation formula $\left[H K_{1}, 2.4\right]$

$$
\int_{\Omega} \sigma \cdot \nabla \zeta d x+\int_{\Omega} \sigma \cdot \xi\left|D^{s} u\right|=\int_{\Omega} f \zeta d x
$$

where $\sigma=\sigma(u)$ and $\xi$ is the Radon-Nikodym derivative of $D^{s} \zeta$ with respect to $\left|D^{s} u\right|$. Indeed, usually we shall write (1.2) as

$$
\int_{\Omega} \sigma \cdot D \zeta d x=\int_{\Omega} f \zeta d x
$$


The absolute continuity (1.1) is necessary for the validity of (1.3) as a simple example illustrates. One may take $n=1, \Omega=(0,3)$, $u \equiv 0$, and $\zeta=\chi_{(1,2)}=$ characteristic function of $(1,2)$ to see that $\left.\int_{\Omega} \phi(D u+t \zeta)\right)=2|t|$ is not differentiable at $t=0$ so that the first variation formula makes no sense.

A local variational principle follows from (1.1) for the functional

$$
I_{r, a}(v)=\int_{\mathrm{B}_{r(a)}} \phi(D v)-\int_{\partial \mathrm{B}_{r}(a)} \sigma \cdot \frac{x}{|x|} v d S-\int_{\mathrm{B}_{r}(a)} f v d x .
$$

Proposition 1.1 The function $u \in B V(\Omega)$ is a local solution if and only if

$$
I_{r, a}(u) \leq I_{r, a}(v)
$$

for every $v \in B V(\Omega)$ and $r<\operatorname{dist}(a, \partial \Omega)$. Moreover,

$$
I_{r, a}(u)=-\int_{\mathrm{B}_{r}(a)} \phi^{*}(\sigma) d x .
$$

Here and in the sequel, $\sigma=\sigma(u)$ where $u$ is the local solution under discussion.

The proof is facilitated by an elementary lemma.

Lemma 1.2. If $v \in B V(\Omega)$ and $D^{s} v \ll\left|D^{s} u\right|$, then

$$
\int_{\mathrm{B}_{r}(a)} \sigma \cdot D v=\int_{\partial \mathrm{B}_{r}(a)} \sigma \cdot \frac{x}{|x|} v d S+\int_{\mathrm{B}_{r}(a)} f v d x .
$$
where

Proof. We assume $a=0$. For fixed $0<\rho<r$, choose $\zeta=\eta$

$$
\eta(x)= \begin{cases}1 & \text { in } \mathbf{B}_{\rho} \\ (r-\rho)^{-1}(r-|x|) & \text { in } \mathbf{B}_{r} \sim \mathbf{B}_{\rho} \\ 0 & \text { in } \Omega \sim \mathbf{B}_{r} .\end{cases}
$$

By (1.1),

$$
\begin{aligned}
\int_{\mathrm{B}_{r}} \eta \sigma \cdot D v & =(r-\rho)^{-1} \int_{\mathrm{B}_{r} \sim \mathrm{B}_{\rho}} \sigma \cdot \frac{x}{|x|} v d x= \\
& =(r-\rho)^{-1}\left\{\int_{\mathrm{B}_{r}} \sigma \cdot \frac{x}{|x|} v d x-\int_{\mathrm{B}_{\rho}} \sigma \cdot \frac{x}{|x|} v d x\right\} .
\end{aligned}
$$


Then, letting $\rho \rightarrow r$, the conclusion follows.

Proof of Proposition 1.1. Suppose first that $u$ is a local solution and that $v \in C^{1}(\Omega)$; hence, $D^{s} v=0 \ll\left|D^{s} u\right|$. By (0.2) and Lemma 1.2,

$$
\begin{aligned}
\int_{\mathrm{B}_{r}(a)} \phi(D v)-\int_{\mathrm{B}_{r}(a)} \phi(D u)= \\
\quad=\int_{\mathbf{B}_{r}(a)}(\phi(\nabla v)-\phi(\nabla u)) d x-\int_{\mathrm{B}_{r}(a)}\left|D^{s} u\right| \\
\geq \int_{\mathbf{B}_{r}(a)} \sigma \cdot(\nabla v-\nabla u) d x-\int_{\mathrm{B}_{r}(a)}\left|D^{s} u\right| \\
=\int_{\mathbf{B}_{r}(a)} \sigma \cdot D(v-u)=\int_{\partial \mathbf{B}_{r}(a)} \sigma \cdot \frac{x}{|x|}(v-u) d S .
\end{aligned}
$$

Thus $I_{r, a}(u) \leq I_{r, a}(v)$ for $v \in C^{1}(\Omega)$.

For an arbitrary $v \in B V(\Omega)$, we may find a sequence $v_{k} \in C^{1}(\Omega)$ such that, for almost every $r$,

$$
\limsup _{k \rightarrow \infty} \int_{\mathbf{B}_{r}} \phi\left(D v_{k}\right) \leq \int_{\mathbf{B}_{r}} \phi(D v) \text { and } v_{k} \rightarrow v \text { in } L^{1}\left(\partial \mathbf{B}_{r}\right) .
$$

Conversely, suppose that (1.3) holds. If $\zeta \in B V(\Omega)$ has $D^{s} \zeta \ll$ $\left|D^{s} u\right|$ and $\operatorname{supp} \zeta \subset \mathrm{B}_{r}(a) \subset \Omega$, then

$$
\int_{\mathrm{B}_{r}(a)} \phi(D(u+\zeta))-\int_{\mathrm{B}_{r}(a)} \phi(D u)=I_{r, a}(u+\zeta)-I_{r, a}(u) \geq 0
$$

hence, $\int_{\Omega} \sigma \cdot D \zeta d x=0$ by our previous reasoning. For an arbitrary $\zeta \in B V_{0}(\Omega)$ with $D^{s} \zeta \ll\left|D^{s} u\right|$, we may use a partition of unity to deduce the equation $\int_{\Omega} \sigma \cdot D \zeta d x=0$ and then obtain from convexity the desired inequality

$$
\int_{\Omega} \phi(D u)=\int_{\Omega} \phi(D u)+\int_{\Omega} \sigma \cdot D \zeta \leq \int_{\Omega} \phi(D u+D \zeta) .
$$

The final assertion, the duality relation localized, is a direct calculation. 
The duality relation is a useful test for minima.

Theorem 1.3 (Monotonicity). Let $f \in L^{\infty}(\Omega)$ and let $u$ be a local solution.

(i) Then,

$$
\rho^{1-n} \int_{\mathbf{B}_{\rho}(a)}|D u| \leq e^{\Lambda(r-\rho)} r^{1-n} \int_{\mathbf{B}_{r}(a)}|D u|+C_{n}(r-\rho),
$$

for $0<\rho<r<\operatorname{dist}(a, \partial \Omega)$, where $\Lambda=C_{n}\|f\|_{L^{\infty}(\Omega)}$ and $C_{n}$ is a dimensional constant.

(ii) If $u \in L^{\infty}(\Omega)$, then

$$
\begin{aligned}
\rho^{1-n} \int_{\mathrm{B}_{\rho}(a)}|D u| \leq e^{\Lambda(r-\rho)} r^{1-n} \int_{\mathrm{B}_{r}(a)}|D u|+ \\
+C_{n}\left(\lambda+\|f\|_{L^{\infty}(\Omega)}\|u\|_{L^{\infty}(\Omega)}\right)(r-\rho),
\end{aligned}
$$

(iii) In particular, the $(n-1)$ density

$$
\Theta(a)=\lim _{r \rightarrow 0} r^{1-n} \int_{\mathrm{B}_{r}(a)}|D u|
$$

exists, is finite, and is uniformly bounded on compact subset of $\Omega$.

We remark immediately that we prove in the next section that any local solution is locally bounded, so that (ii) always holds on compact subset of $\Omega$.

Proof. We again set $a=0$. For $0<r<\operatorname{dist}(0, \partial \Omega)$, define

$$
w(x)= \begin{cases}u\left(r \frac{x}{|x|}\right) & \text { in } \mathbf{B}_{r} \\ u(x) & \text { in } \Omega \sim \mathbf{B}_{r} .\end{cases}
$$

For this $r$, we infer that

$$
I_{r, 0}(u) \leq I_{r, 0}(w) .
$$

Combining this with (0.2) gives that

$$
\begin{aligned}
\int_{\mathbf{B}_{r}}|D u| & \leq \int_{\mathbf{B}_{r}} \phi(D u)+\lambda\left|\mathbf{B}_{r}\right| \\
& \leq \int_{\mathbf{B}_{r}} \phi(D w)-\int_{\mathbf{B}_{r}} f(w-u) d x+\lambda\left|\mathbf{B}_{r}\right| \\
& \leq \int_{\mathbf{B}_{r}}|D w|-\int_{\mathbf{B}_{r}} f(w-u) d x+\lambda\left|\mathbf{B}_{r}\right| .
\end{aligned}
$$


Now by a well-known calculation, cf.[DCP] for example,

$$
\int_{\mathrm{B}_{r}}|D w| \leq(n-1)^{-1} r \int_{\partial \mathrm{B}_{r}}|D u| \text {. }
$$

Now we may estimate the middle term in (1.7) two ways. If $u$ is assumed bounded, then

$$
\left|\int_{\mathbf{B}_{r}} f(w-u) d x\right| \leq 2\|f\|_{L^{\infty}}\|u\|_{L^{\infty}}\left|\mathbf{B}_{r}\right|
$$

Thus

$$
\int_{\mathrm{B}_{r}}|D u| \leq(n-1)^{-1} r \int_{\partial \mathrm{B}_{r}}|D u|+C_{n}\left(\lambda+\|f\|_{L^{\infty}}\|u\|_{L^{\infty}}\right) r^{n}
$$

or

$$
(d / d r)\left\{r^{1-n} \int_{\mathrm{B}_{r}}|D u|+C_{n}\left(\lambda+\|f\|_{L^{\infty}}\|u\|_{L^{\infty}}\right) r\right\} \geq 0
$$

which implies (ii).

If we do not assume that $u$ is bounded, them, since $w-u=0$ on $\partial \mathbf{B}_{r}$,

$$
\begin{aligned}
\left|\int_{\mathrm{B}_{r}} f(w-u) d x\right| & \leq\|f\|_{L^{\infty}}\|w-u\|_{L^{1}\left(\mathrm{~B}_{r}\right)} \\
& \leq C_{n} r\|f\|_{L^{\infty}}\left(\int_{\mathrm{B}_{r}}|D u|+\int_{\mathrm{B}_{r}}|D w|\right)
\end{aligned}
$$

from which (i) follows.

Corollary 1.4. The density $\Theta(x)$ is upper semi-continuous on $\Omega$.

Proof. We assume that $\mathbf{B}_{2 r} \subset \Omega$ and prove upper semi-continuity at 0 . First note that $\mathbf{B}_{\rho}(x) \subset \mathbf{B}_{2 r}$ whenever $x \in \mathbf{B}_{r}$ and $0<\rho<r$. Thus by 1.3

$$
\begin{aligned}
\Theta(x) & \leq \rho^{1-n} \int_{\mathbf{B}_{\rho}(x)}|D u|+C_{n} \rho \\
& \leq \rho^{1-n}(\rho+|x|)^{n-1}(\rho+|x|)^{1-n} \int_{\mathbf{B}_{\rho+|x|}}|D u|+C_{n}(\rho+|x|) \\
& =\left(1+\rho^{-1}|x|\right)^{n-1}(\rho+|x|)^{1-n} \int_{\mathbf{B}_{\rho+|x|}}|D u|+C_{n}(\rho+|x|) .
\end{aligned}
$$


Holding $\rho$ fixed and letting $x \rightarrow 0$ yields that

$$
\limsup _{x \rightarrow 0} \Theta(x) \leq \rho^{1-n} \int_{\mathbf{B}_{\rho}}|D u|+C_{n} \rho .
$$

Then letting $\rho \rightarrow 0$ gives

$$
\limsup _{x \rightarrow 0} \Theta(x) \leq \Theta(0) .
$$

One may easily derive alternative formulas for the density based on the local functional (1.2) and the minimality of $u$.

Corollary 1.5. For $u$ as above and $a \in \Omega$,

$$
\begin{aligned}
\Theta(a) & =\lim _{r \rightarrow 0} r^{1-n} \int_{\mathrm{B}_{r}(a)} \phi(D u) \\
& =\lim _{r \rightarrow 0} r^{1-n} \int_{\partial \mathrm{B}_{r}(a)} \sigma \cdot \frac{x}{|x|} u d S=\lim _{r \rightarrow 0} r^{1-n} \int_{\mathrm{B}_{r}(a)} \sigma \cdot D u .
\end{aligned}
$$

Proof. By (0.1),

$$
0 \leq r^{1-n} \int_{\mathbf{B}_{r}(a)}|D u|-r^{1-n} \int_{\mathbf{B}_{r}(a)} \phi(D u) \leq \lambda r^{1-n}\left|\mathbf{B}_{r}\right| \rightarrow 0
$$

as $r \rightarrow 0$, which implies the first equality. By (1.2),(1.4), and the boundedness of $\sigma$,

$$
\begin{gathered}
r^{1-n} \int_{\mathrm{B}_{r}(a)} \phi(D u)-r^{1-n} \int_{\partial \mathrm{B}_{r}(a)} \sigma \cdot \frac{x}{|x|} u d S= \\
=-\frac{1}{2} r^{1-n} \int_{\mathrm{B}_{r}(a)} \phi^{*}(\sigma) d x \rightarrow 0
\end{gathered}
$$

as $r \rightarrow 0$, since $0 \leq \phi^{*}(\sigma) \leq \lambda$, which implies the second equality. The third equality follows from Lemma 1.2 applied with $v=u$.

Recall that a measurable function $u$ is approximately continuous at $a$ if $a$ is a Lebesgue point for $u$, that is, if

$$
\lim _{\rho \rightarrow 0} \rho^{-n} \int_{\mathbf{B}_{\rho}(a)}|u-c| d x=0 \text { for some } c \in \mathbf{R} .
$$


Corollary 1.6. If a local solution is approximately continuous at $a$, then $\Theta(a)=0$.

Proof. We apply Lemma 1.2 with $v=\eta \cdot(u-c)$ where $c$ is as above and $\eta$ is as in the proof of Lemma 1.2 with $r=2 \rho$. We conclude that

$$
0=\int_{\mathrm{B}_{2 \rho}(a)} \sigma \cdot D v=\int_{\mathrm{B}_{2 \rho}(a)} \sigma \cdot[\eta D u+(u-c) \nabla \eta d x]
$$

hence,

$$
\int_{\mathbf{B}_{\rho}(a)}|D u| \leq \rho^{-1} \int_{\mathbf{B}_{2 \rho}(a)}|u-c| d x .
$$

By 1.5 ,

$$
\begin{aligned}
\Theta(a) & =\lim _{\rho \rightarrow 0} \rho^{1-n} \int_{\mathrm{B}_{\rho}(a)} \sigma \cdot D u \leq \lim _{\rho \rightarrow 0} \rho^{1-n} \int_{\mathbf{B}_{\rho}(a)}|D u| \\
& \leq \lim _{\rho \rightarrow 0} \rho^{-n} \int_{\mathrm{B}_{2_{\rho}}(a)}|u-c| d x=0 .
\end{aligned}
$$

2. The local boundedness. We establish here that a local solution is locally bounded and that it is continuous at each point $a \in \Omega$ where the density $\Theta(a)=0$. Our first step is to estimate the measure of the set where a local solution exceeds a given value. We assume that

$u$ is a local solution in $\Omega$ with $f \in L^{\infty}(\Omega), 0 \in \Omega$, and $\theta: \mathbf{R} \rightarrow \mathbf{R}$ is a bounded increasing piecewise differentiable function with $\theta(t) \leq 1$ for almost all $t$.

Suppose that $0<\rho<r<\min \{1, \operatorname{dist}(0, \partial \Omega)\}$ and let

$$
\eta(x)= \begin{cases}1 & \text { in } \mathbf{B}_{\rho} \\ (r-\rho)^{-1}(r-|x|) & \text { in } \mathbf{B}_{r} \sim \mathbf{B}_{\rho} \\ 0 & \text { in } \Omega \sim \mathbf{B}_{r},\end{cases}
$$

exactly as in Lemma 1.2. We may apply the variational formula (1.1) with $\zeta=\eta \theta(u-c)$, where $c$ is a constant, to obtain that

$$
\begin{aligned}
& \int_{\mathrm{B}_{r}} \eta \sigma \cdot D[\theta(u-c)]= \\
& =(r-\rho)^{-1} \int_{\mathrm{B}_{r} \sim \mathrm{B}_{\rho}} \sigma \cdot \frac{x}{|x|} \theta(u-c) d x+\int_{\mathrm{B}_{r}} \eta \theta(u-c) f d x .
\end{aligned}
$$


Observe that

$$
D[\theta(u-c)]=\theta^{\prime}(u-c) D u \quad \text { and } \quad|D \theta(u-c)|=\theta^{\prime}(u-c)|D u|
$$

Thus, by (0.2) and (0.8),

$$
\begin{aligned}
& \int_{\mathbf{B}_{r}} \eta|D[\theta(u-c)]| \leq \int_{\mathbf{B}_{r}} \eta \theta^{\prime}(u-c)|D u| \\
& \leq \int_{\mathbf{B}_{r}} \eta \theta^{\prime}(u-c) \phi(D u)+\lambda \int_{\mathrm{B}_{r}} \eta \theta^{\prime}(u-c) d x \\
& \leq \int_{\mathbf{B}_{r}} \eta \theta^{\prime}(u-c) \sigma \cdot D u+\lambda \int_{\mathrm{B}_{r}} \eta \theta^{\prime}(u-c) d x \\
&=\int_{\mathbf{B}_{r}} \eta \sigma \cdot D[\theta(u-c)]+\lambda \int_{\mathrm{B}_{r}} \eta \theta^{\prime}(u-c) d x
\end{aligned}
$$

Applying (2.1) and recalling that $|\sigma| \leq 1$, we now obtain the basic estimate

$$
\begin{aligned}
\int_{\mathrm{B}_{\rho}} \eta|D[\theta(u-c)]| & \leq(r-\rho)^{-1} \int_{\mathrm{B}_{r} \sim \mathrm{B}_{\rho}}|\theta(u-c)| d x+\lambda|A| \\
& +\|f\|_{L^{\infty}\left(\mathrm{B}_{r}\right)} \int_{\mathrm{B}_{r}}|\theta(u-c)| d x
\end{aligned}
$$

where $A=\operatorname{supp} \eta \theta(u-c)$.

Now we let $0<h<k<\infty$ and choose

$$
\theta(t)= \begin{cases}0 & \text { for } t \leq k \\ t-k & \text { for } k<t<h \\ h-k & \text { for } t \geq k\end{cases}
$$

Note that

$$
\operatorname{supp}(\eta \theta(u-c)) \subset \mathrm{B}_{r} \cap\{u-c \geq k\}=A(k, r)
$$

Thus (2.2) implies that

$$
\begin{gathered}
\int_{\mathrm{B}_{\rho}}|D[\theta(u-c)]| \leq\left(C+(r-\rho)^{-1}\right) \int_{\mathrm{B}_{\rho}}|\theta(u-c)| d x+\lambda|A(k, r)| \\
C=\|f\|_{L^{\infty}(\Omega)}
\end{gathered}
$$


Let $0<r_{0}<\operatorname{dist}(0, \partial \Omega)$ be given and choose $k_{0}$ so large that

$$
\left|A\left(k_{0}, \rho\right)\right| \leq \frac{1}{2}\left|\mathrm{~B}_{\rho}\right| \text { for } \frac{1}{2} r_{o} \leq \rho \leq r_{0} .
$$

Now $A(k, \rho) \subset A\left(k_{0}, \rho\right)$ for $k>k_{0}$, so by the isoperimetric inequality, the definition of $\theta$, and (2.4),

$$
\begin{gathered}
(h-k)|A(h, \rho)|^{\frac{n-1}{n}} \leq\left(\int_{\mathrm{B}_{\rho}}|\theta(u-c)|^{\frac{n}{n-1}} d x\right)^{\frac{n-1}{n}} \\
\leq c_{0} \int_{\mathrm{B}_{\rho}}|D[\theta(u-c)]| \\
\leq c_{0}\left[C+(r-\rho)^{-1}\right] \int_{\mathrm{B}_{r} \sim \mathrm{B}_{\rho}}|\theta(u-c)| d x+c_{0} \lambda|A(k, r)| \\
\leq c_{0}\left[C+(r-\rho)^{-1}\right](h-k)|A(k, r)|+c_{0} \lambda|A(k, r)| .
\end{gathered}
$$

Hence,

$$
|A(h, \rho)|^{\frac{n-1}{n}} \leq c_{0}(C+\lambda)\left[1+(r-\rho)^{-1}+(h-k)^{-1}\right]|A(k, r)| .
$$

Recalling our restriction that $r_{0}<1$, we may rewrite the above as

$$
\begin{gathered}
|A(h, \rho)|^{\frac{n-1}{n}} \leq \Lambda\left[(r-\rho)^{-1}+(h-k)^{-1}\right]|A(k, r)| \\
\quad \text { for } \frac{1}{2} r_{0} \leq \rho<r \leq r_{0} \text { and } h>k \geq k_{0},
\end{gathered}
$$

where the $\Lambda=\nu_{0}+\nu_{1}\|f\|_{L^{\infty}(\Omega)}$ for suitable constants $\nu_{0}, \nu_{1}$.

The truncation estimate (2.5) differs from the more traditional one of De Giorgi and Stampacchia, cf. for a typical example [KS] p.63, in several notable ways. The first is the sum occuring on the right hand side which is usually a product. The second is the limitation on the range of $\rho$ for which the estimate is valid.

At this point, we state our truncation lemma.

Lemma 2.1. Suppose that $\left\{A(k, r): \frac{1}{2} r_{0} \leq r \leq r_{0}, k \geq k_{0}\right\}$ is any collection of subsets of $\mathbf{B}_{r_{0}}$ that satisfies (2.5) and that $\delta$ is a positive number with

$$
\delta \leq \frac{1}{2}|\mathbf{B}| \text { and } \delta^{\frac{1}{n}} \leq c_{1}^{-1} \text { where } c_{1}=2^{2 n+3} c_{0}(1+\lambda) .
$$


if

$$
\left|A\left(k_{0}, r_{0}\right)\right| \leq \delta r_{0}^{n}
$$

then

$$
\left|A\left(k_{0}+d, \frac{1}{2} r_{0}\right)\right|=0 \text { for } d=c_{1}\left|A\left(k_{o}, r_{0}\right)\right|^{\frac{1}{n}}
$$

Proof. For $i=1,2, \ldots$, let

$$
r_{i}=\frac{1}{2} r_{0}+2^{-i} r_{0} \text { and } k_{i}=k_{0}+d-2^{-i} d
$$

Then

$$
r_{i}-r_{i+1}=2^{-i-1} r_{0} \text { and } k_{i+1}-k_{i}=2^{-i-1} d .
$$

With

$$
\alpha_{i}=\left|A\left(k_{i}, r_{i}\right)\right|^{\frac{n-1}{n}},
$$

our goal is to prove inductively the estimate

$$
\alpha_{i} \leq 2^{-n i} \alpha_{1}
$$

For this, we first note that

$$
\alpha_{1} \leq \delta^{\frac{n-1}{n}} r_{0}^{n-1} \text { and } d=\alpha_{1}^{\frac{1}{n-1}}
$$

Written in terms of the sequence $\alpha_{i}$, the recursion relation (2.5) is

$$
\alpha_{i+1} \leq 2^{i+1} c_{0}\left(r_{0}^{-1}+d^{-1}\right) \alpha_{i}^{\frac{n}{n-1}} .
$$

In particular, by the choise of $\delta$ and $d$,

$$
\begin{aligned}
\alpha_{2} & \leq 4 c_{0}\left(\alpha_{1}^{\frac{1}{n-1}} r_{0}^{-1}+\alpha_{1}^{\frac{1}{n-1}} d^{-1}\right) \alpha_{1} \\
& \leq 4 c_{0}\left(\delta^{\frac{1}{n}}+c^{-1}\right) \alpha_{1} \\
& \leq\left(2^{-2 n-1}+2^{-2 n-1}\right) \alpha_{1}=2^{-2 n} \alpha_{1} .
\end{aligned}
$$


Now assume inductively that $\alpha_{i} \leq 2^{-i n} \alpha_{1}$. Then, by (2.9),

$$
\begin{aligned}
\alpha_{i+1} & \leq 2^{i+1} c_{o}\left(r_{0}^{-1}+d^{-1}\right) \alpha_{1}^{\frac{n}{n-1}}\left(\alpha_{i} \alpha_{1}^{-1}\right)^{\frac{n}{n-1}} \\
& \leq 2^{i} 4 c_{0}\left(\alpha_{1}^{\frac{1}{n-1}} r_{0}^{-1}+\alpha_{1}^{\frac{1}{n-1}} d^{-1}\right) \alpha_{1}\left(2^{-n i}\right)^{\frac{n}{n-1}} \\
& \leq 2^{i} 2^{-2 n}\left(2^{-n i}\right)^{\frac{n}{n-1}} \alpha_{1} \leq 2^{-(i+1) n}
\end{aligned}
$$

the latter inequality holding because the inequality

$$
i-2 n-n i\left(\frac{n}{n-1}\right) \leq-(i+1) n
$$

reduces to simply $n(n-1)+i \geq 0$. This completes the inductive proof of (2.8). The lemma now follows because

$$
\left|A\left(k_{0}+d, \frac{1}{2} r_{0}\right)\right|=\lim _{i \rightarrow \infty} \alpha_{i}^{\frac{n}{n-1}}=0 .
$$

Note the hypotheses of the lemma implies that

$$
d=c_{1}\left|A\left(k_{0}, r_{0}\right)\right|^{\frac{1}{n}} \leq c_{1} \delta^{\frac{1}{n}} r_{0} .
$$

Consequently, whenever (2.5) and (2.7) are satisfied for a local solution $u$ on $\mathbf{B}_{r_{0}}$,

$$
u-c \leq k_{0}+c_{1} \delta^{\frac{1}{n}} \text { in } \mathrm{B}_{r_{0} / 2} .
$$

Since we may argue analogously with

$$
\theta(t)= \begin{cases}h-k & \text { for } t \leq k \\ k-t & \text { for } k<t<h \\ 0 & \text { for } t \geq h\end{cases}
$$

we may conclude that

$$
\|u-c\|_{L^{\infty}}\left(\mathbf{B}_{r_{o} / 2}\right) \leq k_{0}+c_{1} \delta^{1 / n} r_{0}
$$

whenever (2.5) and (2.7) are satisfied for the sets

$$
A(k, r)=\mathbf{B}_{r} \cap\{u \geq k\} \text { and } \tilde{A}(k, r)=\mathbf{B}_{r} \cap\{u \leq k\} .
$$


Theorem 2.2. If $u$ is a local solution in $\Omega$ and $\mathbf{B}_{2 r}(a) \subset \subset \Omega$, then there is a positive constant $M$ depending only on $\|f\|_{L^{\infty}}$ so that

$$
\left\|u-\bar{u}_{r}\right\|_{L^{\infty}\left(\mathrm{B}_{r}(a)\right)} \leq M\left\{r^{1-n} \int_{\mathrm{B}_{2 r}(a)}|D u|+r\right\}
$$

where

$$
\bar{u}_{r}=\left|\mathrm{B}_{r}\right|^{-1} \int_{\mathrm{B}_{r}(a)} u d x .
$$

Proof. We may assume that $a=0$. We may also apply (2.11) to $u-\bar{u}_{r}$, with $r_{0}=2 r$. By (2.5) and (2.11) it only remains to estimate $k_{0}$.

Suppose that $r \leq \rho \leq 2 r<1$. Then we may average the inequality

$$
\left|\bar{u}_{r}-\bar{u}_{\rho}\right| \leq\left|u(x)-\bar{u}_{r}\right|+\left|u(x)-\bar{u}_{\rho}\right|
$$

over the smaller ball $\mathbf{B}_{r}$ to obtain

$$
\begin{aligned}
& \left|\bar{u}_{r}-\bar{u}_{\rho}\right| \leq\left|\mathbf{B}_{r}\right|^{-1} \int_{\mathbf{B}_{r}}\left|u-\bar{u}_{r}\right| d x+\left|\mathbf{B}_{r}\right|^{-1}\left|\mathbf{B}_{\rho}\right|\left|\mathbf{B}_{\rho}\right|^{-1} \int_{\mathbf{B}_{r}}\left|u-\bar{u}_{\rho}\right| d x \\
& \quad \leq c_{2}\left\{r^{1-n} \int_{\mathrm{B}_{r}}|D u|+2^{n} \rho^{1-n} \int_{\mathbf{B}_{\rho}}|D u|\right\} \\
& \quad \leq c_{2}\left\{\left(1+2^{n}\right)^{\Lambda r} \rho^{1-n} \int_{\mathbf{B}_{\rho}}|D u|+c_{n} \rho\right\} \\
& \leq c_{3}\left\{\rho^{1-n} \int_{\mathrm{B}_{\rho}}|D u|+\rho\right\},
\end{aligned}
$$

where we have employed a Poincaré inequality and the monotonicity inequality (Theorem 1.3).

Letting

$$
A_{r}(k, \rho)=\mathrm{B}_{\rho} \cap\left\{\left|u-\bar{u}_{r}\right| \geq k\right\},
$$

we use a Sobolev inequality and (2.13) to calculate

$$
\begin{aligned}
k\left|A_{r}(k, \rho)\right|^{\frac{n-1}{n}} & \leq\left\|u-\bar{u}_{r}\right\|_{L^{\frac{n}{n-1}}\left(\mathbf{B}_{\rho}\right)} \\
& \leq\left\|u-\bar{u}_{\rho}\right\|_{L^{\frac{n}{n-1}}\left(\mathrm{~B}_{\rho}\right)}+\left\|\bar{u}_{\rho}-\bar{u}_{r}\right\|_{L^{\frac{n}{n-1}}\left(\mathbf{B}_{\rho}\right)} \\
& \leq c_{4} \int_{\mathrm{B}_{\rho}}|D u|+c_{3}\left\{\rho^{1-n} \int_{\mathrm{B}_{\rho}}|D u|+\rho\right\}|\mathbf{B}|^{\frac{n-1}{n}}
\end{aligned}
$$


or

$$
k\left(\rho^{-n}\left|A_{r}(k, \rho)\right|\right)^{\frac{n-1}{n}} \leq c_{5}\left\{\rho^{1-n} \int_{\mathrm{B}_{\rho}}|D u|+\rho\right\} .
$$

Selecting

$$
k_{0}=\inf \left\{\ell:\left|A_{r}(k, \rho)\right| \leq \delta \rho^{n} \text { for all } r \leq \rho \leq 2 r, k \geq \ell\right\}
$$

now permits us to apply (2.11) with $r_{0}=2 r$ as well as to estimate

$$
\begin{aligned}
k_{0} & \leq c_{5} \delta^{\frac{n}{n-1}} \sup _{r \leq \rho \leq 2 r}\left\{\rho^{1-n} \int_{\mathrm{B}_{\rho}}|D u|+\rho\right\} \\
& \leq c_{6}\left\{r^{1-n} \int_{\mathrm{B}_{r}}|D u|+r\right\} .
\end{aligned}
$$

This, combined with (2.11), completes the proof.

Corollary 2.3. The local solution $u$ is continuous at $a \in \Omega$ if and only if

$$
\Theta(a)=\lim _{r \rightarrow 0} r^{1-n} \int_{\mathrm{B}_{r}(a)}|D u|=0
$$

Moreover,

$$
\|u-u(a)\|_{L^{\infty}\left(\mathrm{B}_{r}(a)\right)} \leq N\left\{r^{1-n} \int_{\mathrm{B}_{2} r(a)}|D u|+r\right\}
$$

for some constant $N$ depending only on $\Omega, f$, and $a$.

Proof. We again take $a=0$. The necessity of the condition $\theta(a)=0$ was established in 1.5 . To use 2.2 to prove the sufficiency we need to control the averages $\bar{u}_{\rho}$ as $\rho \rightarrow 0$. Using 2.2 , we see that, for each positive $\rho \leq r$,

$$
\begin{aligned}
\left|\bar{u}_{\rho}\right| & \leq\left|\mathrm{B}_{\rho}\right|^{-1}\left|\int_{\mathbf{B}_{\rho}} u d x\right| \\
& \leq\left|\bar{u}_{r}\right|+\left|\mathrm{B}_{\rho}\right|^{-1} \int_{\mathbf{B}_{\rho}}\left|u-\bar{u}_{r}\right| d x \\
& \leq\left|\bar{u}_{r}\right|+M\left\{r^{1-n} \int_{\mathbf{B}_{2 r}}|D u|+r\right\} .
\end{aligned}
$$


Thus the collection $\left\{\bar{u}_{\rho}: 0<\rho \leq r\right\}$ is uniformly bounded. Moreover,

$$
\begin{aligned}
\left|\bar{u}_{\rho}-\bar{u}_{r}\right| & \leq \\
& \leq\left|\mathrm{B}_{\rho}\right|^{-1} \int_{\mathrm{B}_{\rho}}\left|u-\bar{u}_{\rho}\right| d x+\left|\mathrm{B}_{\rho}\right|^{-1} \int_{\mathrm{B}_{\rho}}\left|u-\bar{u}_{r}\right| d x \\
& \leq c_{2} \rho^{1-n} \int_{\mathrm{B}_{\rho}}|D u|++M\left\{r^{1-n} \int_{\mathrm{B}_{2 r}}|D u|+r\right\} .
\end{aligned}
$$

It follows that $\left\{\bar{u}_{\rho}\right\}$ is Cauchy as $\rho \rightarrow 0$ if $\Theta(0)=0$. In this case, the limit $u(0)$ satisfies

$$
|u-u(0)| \leq N\left\{r^{1-n} \int_{\mathrm{B}_{2 r}}|D u|+r\right\} \text { in } \mathrm{B}_{r} .
$$

3. Points of positive density. From [F], 4.5.9(3) we recall that a BV function $u$ has, at $\mathcal{H}^{n-1}$ almost all points $a$ of its domain, finite approximate upper and lower limits $u^{+}(a) \geq u^{-}(a)$ satisfying

$$
\begin{aligned}
& u^{+}(a)=\inf \left\{k: \lim _{\rho \rightarrow 0^{+}}\left|\mathbf{B}_{\rho}\right|^{-1}\left|\mathbf{B}_{\rho}(a) \cap\{u>k\}\right|=0\right\} \text { and } \\
& u^{-}(a)=\sup \left\{k: \lim _{\rho \rightarrow 0^{+}}\left|\mathbf{B}_{\rho}\right|^{-1}\left|\mathbf{B}_{\rho}(a) \cap\{u<k\}\right|=0\right\} .
\end{aligned}
$$

Moreover, using $[\mathrm{F}], 4.5 .9(27)$, one finds that these limits differ at $\mathcal{H}^{n-1}$ almost all points a where the upper $n-1$ density

$$
\limsup _{r \rightarrow 0} r^{1-n} \int_{\mathrm{B}_{r}(a)}|D u|>0 .
$$

In this section we shall limit ourselves to the case where $f=0$.

Theorem 3.1. Suppose that $f=0$ and $u$ is a local solution in $\Omega, a \in \Omega, \Theta(a)>0$ and $u^{+}>u^{-}$where $u^{ \pm}=u^{ \pm}(a)$. For any $\epsilon>0$, there is a $\sigma>0$ so that

$$
u^{-}-\epsilon<u<u^{+}+\epsilon \text { in } \mathrm{B}_{\sigma}(a) .
$$


Proof. We assume $a=0$. For fixed $0<\rho<r<\operatorname{dist}(0, \partial \Omega)$, we again let $\eta$ be as in 1.2, and, for fixed $h>k \geq 0$, let $\theta$ be as in (2.3). We now use the test function

$$
\zeta(x)=\eta(x) \cdot \theta\left(u(x)-u^{+}\right)
$$

in the variational formula (1.1). Since

$$
\operatorname{supp} \zeta \subset \mathbf{B}_{r} \cap \operatorname{supp}\left(\theta\left(u-u^{+}\right)\right)=\mathbf{B}_{r} \cap\left\{u-u^{+} \geq k\right\}
$$

we deduce, exactly as in the proof of (2.4) and using that $u$ is bounded, that

$$
\int_{\mathrm{B}_{\rho}}\left|D\left[\theta\left(u-u^{+}\right)\right]\right| \leq(r-\rho)^{-1} \int_{\mathrm{B}_{r} \sim \mathrm{B}_{\rho}}\left|\theta\left(u-u^{+}\right)\right| d x+C\left|A^{+}(k, r)\right|
$$

where $C$ is a constant and

$$
A^{+}(k, r)=\mathbf{B}_{r} \cap\left\{u-u^{+} \geq k\right\}
$$

By (3.1), we may, for any positive number $k_{0}$, choose a positive $r_{0}<\min \left\{k_{0}, \operatorname{dist}(0, \partial \Omega)\right\}$ so that

$$
\left|A^{+}\left(k_{0}, r_{0}\right)\right| \leq \min \left\{\delta, 2^{-n-1}|\mathrm{~B}|\right\} r_{0}^{n}
$$

where $\delta$ is the constant from Lemma 2.1. Then, for $\frac{1}{2} r_{0} \leq \rho \leq r_{0}$ and $k \geq k_{0}$,

$$
\left|A^{+}(k, \rho)\right| \leq\left|A^{+}\left(k_{0}, r_{0}\right)\right| \leq\left. 2^{-n-1}\left|\mathbf{B}_{r_{0}}\right| \mathbf{B}_{\rho}\right|^{-1}\left|\mathbf{B}_{\rho}\right| \leq \frac{1}{2}\left|\mathbf{B}_{r_{0}}\right| .
$$

Since

$$
\mathbf{B}_{\rho} \cap \operatorname{supp}\left(\theta\left(u-u^{+}\right)\right) \subset\left|A^{+}(k, \rho)\right|,
$$

we infer from a Sobolev inequality and (3.2) that, for $\frac{1}{2} r_{0} \leq \rho<r \leq$ $r_{0}$ and $h>k \geq k_{0}$,

$$
(h-k)\left|A^{+}(h, \rho)\right|^{\frac{n-1}{n}} \leq\left[\int_{A^{+}(h, \rho)}\left|\theta\left(u-u^{+}\right)\right|^{\frac{n}{n-1}} d x\right]^{\frac{n-1}{n}} \leq
$$




$$
\begin{aligned}
& \leq\left[\int_{\mathrm{B}_{\rho}}\left|\theta\left(u-u^{+}\right)\right|^{\frac{n}{n-1}} d x\right]^{\frac{n-1}{n}} \leq c_{0} \int_{\mathrm{B}_{r}}\left|D\left[\theta\left(u-u^{+}\right)\right]\right| \\
& \leq c_{0}(r-\rho)^{-1} \int_{\mathrm{B}_{r} \sim \mathrm{B}_{\rho}}\left|\theta\left(u-u^{+}\right)\right| d x+c_{0} C\left|A^{+}(k, r)\right| \\
& \quad \leq c_{0}(r-\rho)^{-1}(h-k)\left|A^{+}(k, r)\right|+c_{0} C\left|A^{+}(k, r)\right| .
\end{aligned}
$$

Thus we find, as in our previous argument in $\S 2$ that for a constant $C_{0}$,

$$
\begin{array}{r}
\left|A^{+}(h, \rho)\right|^{\frac{n-1}{n}} \leq C_{0}\left[(r-\rho)^{-1}+(h-k)^{-1}\right]\left|A^{+}(k, r)\right| \\
\qquad \text { for } \frac{1}{2} r_{0} \leq \rho<r \leq r_{0} \text { and } h>k \geq k_{0} .
\end{array}
$$

By (3.3) and (3.4), we may again apply Lemma 2.1 to conclude that

$$
u-u^{+} \leq k_{0}+d=k_{0}+c_{1}\left|A\left(k_{0}, r_{0}\right)\right|^{\frac{1}{n}} \leq c_{7} k_{0} \text { in } \mathbf{B}_{r_{o} / 2} .
$$

Similarly, there is a positive $s_{0}$ such that

$$
u-u^{-} \geq c_{7} k_{0} \text { in } \mathbf{B}_{s_{0} / 2}
$$

Next we seek more precise information on the behavior of $u$ near a point of positive density. From $[\mathrm{F}], 4.5 .9(22)$, we recall that, a $B V$ function $u$, is, at $\mathcal{H}^{n-1}$ almost all points $a$ of positive upper density, approximately two-sided continuous in the sense that there is a unit vector $\nu(a)$ so that

$$
\alpha^{ \pm}(\rho)=\left[\rho^{-n} \int_{\mathbf{B}_{\rho}^{+}(a)}\left|u-u^{+}\right|^{\frac{n}{n-1}} d x\right]^{\frac{n-1}{n}} \rightarrow 0 \text { as } \rho \rightarrow 0
$$

where $\mathrm{B}_{\rho}^{ \pm}(a)=\mathrm{B}_{\rho}(a) \cap\{ \pm(x-a) \cdot \nu(a)>0\}$.

Theorem 3.2. Suppose that, in addition to the hypotheses of Theorem 3.1, the local solution $u$ is approximately two-sided continuous at $a$ in the direction $\nu$. For any $0<\beta<\frac{1}{n-1}$ and any $\epsilon>0$, there is a $\tau>0$ so that

$$
\begin{gathered}
\left|u-u^{ \pm}\right| \leq \epsilon \\
\text { in } E^{ \pm}=\mathbf{B}_{\tau}(a) \cap\left\{x: \pm(x-a) \cdot \nu \geq\left[\alpha^{ \pm}(2|x-a|)\right]^{\beta}|x-a|\right\}
\end{gathered}
$$


where $\alpha^{ \pm}$is as above. In particular, the two sets

$$
\left\{x: u(x)>\frac{1}{2}\left(u^{+}+u^{-}\right)\right\} \text {and }\left\{x: u(x)<\frac{1}{2}\left(u^{+}+u^{-}\right)\right\}
$$

contain open domains whose boundaries are tangent to the hyperplane $\{(x-a) \cdot \nu=0\}$ at a. Moreover,

$$
\lim _{\rho \rightarrow 0} \int_{\mathbf{B}_{\rho}(a)} D u / \int_{\mathbf{B}_{\rho}(a)}|D u|=\nu .
$$

Proof. We assume $a=0$. Choose a positive number $s \leq$ $\frac{1}{2}\left(c_{1}^{-1} \delta^{-\frac{1}{n}}\right)$ so that $\mathrm{B}_{s} \subset \Omega$ and

$$
\left[\alpha^{+}(2 t)\right]^{1-\beta(n-1)} \leq 4^{1-n}(\delta|\mathbf{B}|)^{\frac{n-1}{n}} \epsilon \text { for all } 0<t \leq s .
$$

Suppose $b \in \mathbf{B}_{s}$ and $b . \nu \geq\left[\alpha^{+}(2|b|)\right]^{\beta}|b|$. Setting

$$
r_{0}=\left[\alpha^{+}(2|b|)\right]^{\beta}|b| \text { and } k_{0}=\frac{1}{2} \epsilon
$$

we see that

$$
k_{0}^{-1} 4^{n-1}|\mathrm{~B}|^{\frac{n}{n-1}}\left[\alpha^{+}(2|b|)\right]\left(r_{0}^{-1}|b|\right)^{n-1} \leq \delta^{\frac{n-1}{n}} .
$$

Next, for $\frac{1}{2} r_{0} \leq \rho \leq r_{0}$ and $k \geq k_{0}$, we let

$$
A_{b}(k, \rho)=\mathbf{B}_{\rho}(b) \cap\left\{u<u^{+}-k\right\},
$$

note that $\mathbf{B}_{\rho}(b) \subset \mathbf{B}_{\rho+|b|}^{+}$because $b . \nu \geq \rho$, and use the definition of $\alpha^{+}$and (3.7) to estimate

$$
\begin{aligned}
k\left|A_{b}(k, \rho)\right|^{\frac{n-1}{n}} & \leq\left[\int_{A_{b}(k, \rho)}\left|u-u^{+}\right|^{\frac{n}{n-1}} d x\right]^{\frac{n-1}{n}} \\
& \leq\left[\int_{\mathrm{B}_{\rho+|b|}^{+}}\left|u-u^{+}\right|^{\frac{n}{n-1}} d x\right]^{\frac{n-1}{n}} \\
& \leq\left[\alpha^{+}(\rho+|b|)\right](\rho+|b|)^{n-1} \\
& \leq 2^{n-1}\left[\alpha^{+}(2|b|)\right]\left(\rho^{-1}|b|\right)^{n-1} \rho^{n-1} \\
& \leq 4^{n-1}\left[\alpha^{+}(2|b|)\right]\left(r_{0}^{-1}|b|\right)^{n-1} \rho^{n-1} \\
& \leq k_{0} \delta^{\frac{n-1}{\rho}} \rho^{n-1} \leq k \delta^{\frac{n-1}{n}} \rho^{n-1} .
\end{aligned}
$$


Thus,

$$
\left|A_{b}(k, \rho)\right| \leq \delta \rho^{n} \text { for } \frac{1}{2} r_{0} \leq \rho \leq r_{0} \text { and } k \geq k_{0}
$$

Now for fixed $\rho$ and $r$ with $\frac{1}{2} r_{0} \leq \rho<r \leq r_{0}$, we let $\eta$ be as in 1.2. Also for fixed $h$ and $k$ with $h>k \geq k_{0}$, we let

$$
\theta(t)= \begin{cases}h-k & \text { for } t \leq-k \\ -t-k & \text { for }-h<t<-k \\ 0 & \text { for } t \geq-k\end{cases}
$$

Using $\zeta(x)=\eta(x) \cdot \theta\left(u(x)-u^{+}\right)$in the variational formula (1.1) and noting that

$$
\operatorname{supp}\left(\eta \theta^{\prime}(u)\right) \subset \operatorname{supp} \zeta \subset A_{b}(k, r)
$$

we deduce, as in the proof of (2.4), that

$$
\int_{\mathbf{B}_{\rho}}\left|D\left[\theta\left(u-u^{+}\right)\right]\right| \leq(r-\rho)^{-1} \int_{\mathbf{B}_{r} \sim \mathbf{B}_{\rho}}\left|\theta\left(u-u^{+}\right)\right| d x+C\left|A_{b}(k, r)\right| .
$$

Since $\delta \leq \frac{1}{2}|\mathbf{B}|$, we may, as in $\S 2$, use a Sobolev inequality to find that

$$
\left|A_{b}(h, \rho)\right|^{\frac{n-1}{n}} \leq \text { const. }\left[(r-\rho)^{-1}+(h-k)^{-1}\right]\left|A_{b}(k, r)\right|
$$

for $\frac{1}{2} r_{0} \leq \rho<r \leq r_{0}$ and $h>k \geq k_{0}$. Applying 2.1 now gives

$$
\left|A_{b}\left(k_{0}+d, \frac{1}{2} r_{0}\right)\right|=0 \text { where } d=c_{1}\left|A_{b}\left(k_{0}, r_{0}\right)\right|^{\frac{1}{n}}
$$

Thus,

$$
u-u^{+} \geq-\left(k_{0}+c_{1} \delta^{\frac{1}{n}} r_{0}\right) \geq-\left(\frac{1}{2} \epsilon+c_{1} \delta^{\frac{1}{2}} s\right) \geq \epsilon \text { in } \mathrm{B}_{r_{o} / 2}(b)
$$

Similarly, for $b \in \mathbf{B}_{s}$ with $b . \nu \leq-\left[\alpha^{-}(2|b|)\right]^{\beta}|b|$, we use

$$
r_{0}=\left[\alpha^{-}(2|b|)\right]^{\beta}|b|
$$

and find that

$$
u-u^{+} \leq-\epsilon \text { in } \mathbf{B}_{r_{o} / 2}(b)
$$


Combining these two inequalities with Theorem 3.1 gives the existence os a suitable $\tau$.

Finally to obtain the formula for the vector $\nu$, we write

$$
\int_{\mathrm{B}_{\rho}} D u=\int_{\partial \mathrm{B}_{\rho}} u \frac{x}{|x|} d S
$$

where the right hand side is to be understood in the sense of $B V$ trace theory $[G]$, and consider the integrals over the three spherical regions $\partial \mathbf{B}_{\rho} \cap E^{+}, \partial \mathbf{B}_{\rho} \cap E^{-}$, and $\partial \mathbf{B}_{\rho} \sim\left(E^{+} \cup E^{-}\right)$separately.

To handle the last region, note that by Theorem 3.1, $u$ is bounded on $\mathbf{B}_{\rho}$ for $\rho$ sufficiently small. Thus,

$$
\begin{aligned}
& \left|\int_{\partial \mathbf{B}_{\rho} \sim\left(E^{+} \cup E^{-}\right)} u \frac{x}{|x|} d S\right| \leq \\
& \leq\|u\|_{L^{\infty}\left(\mathbf{B}_{\rho}\right)} \mathcal{H}^{n-1}\left(\partial \mathbf{B}_{\rho} \sim\left(E^{+} \cup E^{-}\right)\right) \\
& \leq c\|u\|_{L^{\infty}\left(\mathbf{B}_{\rho}\right)} \max \left\{\left[\alpha^{+}(2 \rho)\right]^{\beta},\left[\alpha^{-}(2|b|)\right]^{\beta}\right\} \rho^{n-1}=o\left(\rho^{n-1}\right) .
\end{aligned}
$$

For the other two regions, we use the first conclusion of 3.2 to see that

$$
\begin{aligned}
\mid \int_{\partial \mathrm{B}_{\rho} \cap E^{ \pm}} u & \frac{x}{|x|} d S-\int_{\partial \mathrm{B}_{\rho} \cap E^{ \pm}} u^{ \pm} \frac{x}{|x|} d S \mid \leq \\
& \leq \int_{\partial \mathrm{B}_{\rho} \cap E^{ \pm}}\left|u-u^{ \pm}\right| d S \\
& \leq \operatorname{ess} \sup _{\partial \mathrm{B}_{\rho} \cap E^{ \pm}}\left|u-u^{ \pm}\right| \mathcal{H}^{n-1}\left(\partial \mathbf{B}_{\rho}\right)=o\left(\rho^{n-1}\right) .
\end{aligned}
$$

Moreover, since each set $E^{ \pm}$is symmetric in the directions orthogonal to $\nu$,

$$
\int_{\partial \mathrm{B}_{\rho} \cap E^{ \pm}} u^{ \pm} \frac{x}{|x|} d S=\left(\int_{\partial \mathrm{B}_{\rho} \cap E \pm} \frac{x}{|x|} \cdot \nu d S\right) u^{ \pm} \nu .
$$

We conclude that

$$
\rho^{1-n} \int_{\mathbf{B}_{\rho}} D u=\gamma \nu+0(1) \text { as } \rho \rightarrow 0,
$$

where $\gamma=\left[\left(\int_{\partial \mathrm{B} \cap E^{+}} \frac{x}{|x|} \cdot \nu d S\right) u^{+}+\left(\int_{\partial B \cap E^{-}} \frac{x}{|x|} \cdot \nu d S\right) u^{-}\right]$is a nonzero scalar. Then

$$
\rho^{1-n} \int_{\mathbf{B}_{\rho}}|D u|=|\gamma|+o(1) \text { as } \rho \rightarrow 0
$$


and we may divide and let $\rho \rightarrow 0$ to obtain the desired formula.

As a consequence of Theorem 3.2 and the characterization from [F], 4.5.9(22), the set $E^{+}$defined in (3.5) is a set of finite perimeter and (3.6) holds on $\partial E^{+} \cap B_{\delta}(a), \delta<\tau, \mathcal{H}^{n-1}$ a.e. Thus for $f=0$ and $\eta$ smooth with supp $\eta \subset B_{\delta}(a)$,

$$
D\left(\eta \chi_{E^{+}}\right) \ll|D u|
$$

Working directly with the variational principle for the functional, this leads in a standard way to

Theorem 3.3 Suppose that $f=0$ and $u$ is a local solution in $\Omega, a \in \Omega, \Theta(a)>0$, and $u^{+}(a)>u^{-}(a)$. Suppose in addition that $u$ is two-sided continuous at a in the direction $\nu$. Then there is a neighborhood $\mathbf{B}_{\tau}($ a) such that the set

$$
\partial\left\{x: u(x)>\frac{1}{2}\left(u^{+}+u^{-}\right)\right\} \cap \mathbf{B}_{\tau}(a)
$$

is an area minimizing hypersurface in $\mathbf{B}_{\tau}(a)$.

We adopt several standard notations: $\mathrm{B}_{r}(a)$ indicates the open ball $\left\{x \in \mathbf{R}^{n}:|x-a|<r\right\}$ and $\mathbf{B}_{r}=\mathbf{B}_{r}(0)$. The Lebesgue outer measure of a set $A$ is written $|A|$, while $\mathcal{H}^{n-1}$ denotes $n-1$ dimensional Hausdorff measure. When restricted to a sphere $\partial \mathbf{B}_{r}(a)$, the latter becomes ordinary surface measure and will be, under integrals signs, abbreviated as $d S$.

This research was supported in part by NSF grants DMS 8511357 and DMS 87-0672. 


\section{References}

[A] G.Anzellotti, On the extremal stress and displacement in Hencky plasticity, Duke Math. J. 551 (1984), 133-147.

[AG $\left.\mathrm{AG}_{1}\right]$ G.Anzellotti and M.Giaquinta, Existence of the displacement field for an elastoplastic body, Manus.Math. 32 (1980), 101-136.

$\left[\mathrm{AG}_{2}\right]$ G.Anzellotti, M.Giaquinta, On the existence of the fields of stress and displacement for an elasto-plastic body in static equilibrium, J.Math. Pures Appl. 61 (1982), 219-244.

[AL] G.Anzellotti, S.Luckhans, Dynamical evolution of elasto-perfectly plastic bodies, Appl. Math. Opt. 15 (1987), 121-140.

$\left[\mathrm{B}_{1}\right]$ H.Brezis, Intégrales convexes dans les espaces de Sobolev, Israel J. Math. 13 (1972), 9-23.

$\left[\mathrm{B}_{2}\right]$ H.Brezis, Multiplicateur de Lagrange en torsion elastoplastique, Arch. Rat. Mech. Anal. 49 (1972), 32-40.

$\left[\mathrm{B}_{3}\right]$ H.Brezis, Analyse fonctionnelle, Masson, (1983).

[DCP] E.De Giorgi,F.Colombini, L.Piccinini, Frontiere orientate di misura minima e questioni collegate, Pubbl. Scuola Normale, Pisa, (1972).

[ET] I.Ekeland, R.Temam, Convex duality, North-Holland, (1976).

[F] H.Federer, Geometric Measure Theory, Springer-Verlag, Berlin, Heidelberg and New York, 1969.

[G] E.Gagliardo, Caratterizzazioni delle tracce sulla frontiera relative ad alcune classi di funzioni in $n$ variabili, Rend. Sem. Mat. Padova 27 (1957), 284-305.

[HK 1 R.Hardt, D.Kinderlehrer, Elastic plastic deformation, Appl. Math. Optim. 10 (1983), 203-246.

$\left[\mathrm{HK}_{2}\right]$ R.Hardt, D.Kinderlehrer, Some regularity results in plasticity, Appl. Symp. Pure Math. 44 (1986), 239-244.

[KS] D.Kinderlehrer, G.Stampacchia, Variational Inequalities, Academic Press, 1980.

[KT] R.Kohn, R.Temam, Dual spaces of stresses and strains, Appl. Math. Optim. 10 (1983), 1-35.

[RS] V.Roytburd and M.Slemrod, Dynamic phase transitions and compensated compactness, J.Bona, C.Dafermos, J.L.Ericksen, D.Kinderlehrer eds., IMA Volumes in Math. and Appl. 4 (1987), 289-304. 
[S] G.Stampacchia, Formes bilinéaires coércitives sur les ensembles convexes, CRAS Paris 258 (1964), 4413-4416.

[T] R.Temam, Plasticity, Gauthier Villars (1983).

School of Mathematics

University of Minnesota 206 Church St. SE

MINNEAPOLIS, MN 55455 
Yisong Yang Existence, Regularity, and Asymptotic Behavior of the Solutions to the Ginzburg-Landau Equations on $\mathbf{R}^{3}$

Chjan. C. Lim On Symplectic Tree Graphs

Wilhelm I. Fushchich, Ivan Krivsky and Vladimir Simulik, On Vector and Pseudovector Lagrangians for Electromagnetic Field

Wilhelm I. Fushchich, Exact Solutions of Multidimensional Nonlinear Dirac's and Schrödinger's Equations

Wilhelm I. Fushchich and Renat Zhdanov, On Some New Exact Solutions of Nonlinear D'Allembert and Hamilton Equations

Brian A. Coomes, The Lorenz System Does Not Have a Polynomial Flow

J.W. Helton and N.J. Young, Approximation of Hankel Operators: Truncation Error in an $H^{\infty}$ Design Method

Gregory Ammar and Paul Gader, A Variant of the Gohberg-Semencul Formula Involving Circulant Matrices

R.L. Fosdick and G.P. MacSithigh, Minimization in Nonlinear Elasticity Theory for Bodies Reinforced with Inextensible Cords

Fernando Reitich, Rapidly Stretching Plastic Jets: The Linearized Problem

Francisco Bernis and Avner Friedman, Higher Order Nonlinear Degenerate Parabolic Equations

Xinfu Chen and Avner Friedman, Maxwell's Equations in a Periodic Structure

Avner Friedman and Michael Vogelius Determining Cracks by Boundary Measurements

Yuji Kodama and John Gibbons, A Method for Solving the Dispersionless KP Hierarchy and its Exact Solutions II

Yuji Kodama, Exact Solutions of Hydrodynamic Type Equations Having Infinitely Many Conserved Densities

Robert Carroll, Some Forced Nonlinear Equations and the Time Evolution of Spectral Data

Chjan. C. Lim Spanning Binary Trees, Symplectic Matrices, and Canonical Transformations for Classical N-body Problems

E.F. Assmus, Jr. and J.D. Key, Translation Planes and Derivation Sets

Matthew Witten, Mathematical Modeling and Computer Simulation of the Aging-Cancer Interface

Matthew Witten and Caleb E. Finch, Re-Examining The Gompertzian Model of Aging

Bei Hu, A Free Boundary Problem for a Hamilton-Jacobi Equation Arising in Ions Etching

T.C. Hu, Victor Klee and David Larman, Optimization of Globally Convex Functions

Pierre Goossens, Shellings of Tilings

D. David, D. D. Holm, and M.V. Tratnik, Integrable and Chaotic Polarization Dynamics in Nonlinear Optical Beams

D. David, D.D. Holm and M.V. Tratnik, Horseshoe Chaos in a Periodically Perturbed Polarized Optical Beam

Laurent Habsieger, Linear Recurrent Sequences and Irrationality Measures

Laurent Habsieger, MacDonald Conjectures and The Selberg Integral

David Kinderlehrer and Giorgio Vergara-Caffarelli, The Relaxation of Functionals with Surface Energies

Richard James and David Kinderlehrer, Theory of Diffusionless Phase Transitions

David Kinderlehrer, Recent Developments in Liquid Crystal Theory

Niky Kamran and Peter J. Olver, Equivalence of Higher Order Lagrangians

1. Formulation and Reduction

Lucas Hsu, Niky Kamran and Peter J. Olver, Equivalence of Higher Order Lagrangians II. The Cartan Form for Particle Lagrangians

D.J. Kaup and Peter J. Olver, Quantization of BiHamiltonian Systems

Metin Arik, Fahrünisa Neyzi, Yavuz Nutku, Peter J. Olver and John M. Verosky Multi-Hamiltonian Structure of the Born-Infeld Equation

David H. Wagner, Detonation Waves and Deflagration Waves in the One Dimensional ZND Model for High Mach Number Combustion

Jerrold R. Griggs and Daniel J. Kleitman, Minimum Cutsets for an Element of a Boolean Lattice

Dieter Jungnickel, On Affine Difference Sets

Pierre Leroux, Reduced Matrices and q-log Concavity Properties of q-Stirling Numbers

A. Narain and Y. Kizilyalli, The Flow of Pure Vapor Undergoing Film Condensation Between Parallel Plates

Donald A. French, On the Convergence of Finite Element Approximations of a Relaxed Variational Problem 
504 Yisong Yang, Computation, Dimensionality, and Zero Dissipation Limit of the Ginzburg-Landau Wave Equation

Jürgen Sprekels, One-Dimensional Thermomechanical Phase Transitions with Non-Convex Potentials of Ginzburg-Landau Type

Yisong Yang, A Note On Nonabelian Vortices

Yisong Yang, On the Abelian Higgs Models with Sources

Chjan. C. Lim, Existence of Kam Tori in the Phase Space of Vortex Systems

John Weiss, Bäcklund Transformations and the Painlevé Property

Pu Fu-cho and D.H. Sattinger, The Yang-Baxter Equation for Integrable Systems

E. Bruce Pitman and David G. Schaeffer, Instability and Ill-Posedness in Granular Flow

Brian A. Coomes, Polynomial Flows on $\mathbb{C}^{n *}$

Bernardo Cockburn, Suchung Hou and Chi-Wang Shu, The Runge-Kutta Local

Projection Discontinuous Galerkin Finite Element Method for Conservation Laws IV:

The Multidimensional Case

514

515

516

517

Peter J. Olver, Invariant Theory, Equivalence Problems, and the Calculus of Variations

Daniel D. Joseph and Thomas S. Lundgren with an appendix by R. Jackson and D.A. Saville, Ensemble Averaged and Mixture Theory Equations

P. Singh, Ph. Caussignac, A. Fortes, D.D. Joseph and T. Lundgren, Stability of Periodic Arrays of Cylinders Across the Stream by Direct Simulation

Daniel D. Joseph, Generalization of the Foscolo-Gibilaro Analysis of Dynamic Waves

A. Narain and D.D. Joseph, Note on the Balance of Energy at a Phase Change Interface

Daniel D. Joseph, Remarks on inertial radii, persistent normal stresses, secondary motions, and non-elastic extensional viscosities

D. D. Joseph, Mathematical Problems Associated with the Elasticity of Liquids

Henry C. Simpson and Scott J. Spector, Some Necessary Conditions at an Internal Boundary for Minimizers in Finite Elasticity

Peter Gritzmann and Victor Klee, On the 0-1 Maximization of Positive Definite Quadratic Forms

Fu-Cho Pu and D.H. Sattinger, The Yang-Baxter Equations and Differential Identities

Avner Friedman and Fernando Reitich, A Hyperbolic Inverse Problem Arising in the Evolution of Combustion Aerosol

E.G. Kalnins, Raphael D. Levine and Willand Miller, Jr., Conformal Symmetries and Generalized Recurrences for Heat and Schrödinger Equations in One Spatial Dimension

Wang Jinghua and Gerald Warnecke, On Entropy Consistency of Large Time Step Godunov and Glimm Schemes

C. Guillopé and J.C. Saut, Existence Results for the Flow of Viscoelastic Fluids with a Differential Constitutive Law

H.L. Bodlaender, P. Gritzmann, V. Klee and J. Van Leeuwen Computational Complexity of Norm-Maximization

Li Ta-tsien (Li Da-qian) and Yu Xin, Life-Span of Classical Solutions to Fully Nonlinear Wave Equations

Jong-Shenq Guo, A Variational Inequality Associated with a Lubrication Problem

Jong-Shenq Guo, On the Semilinear Elliptic Equation $\Delta w-\frac{1}{2} y \cdot \nabla w+\lambda w-w^{-\beta}$ in $R^{n}$

Andrew E. Yagle, Inversion of the Bloch transform in magnetic resonance imaging using asymmetric two-component inverse scattering

Bei Hu, A Fiber Tapering Problem

Peter J. Olver, Canonical Variables for BiHamiltonian Systems

Michael Renardy, A Well-Posed Boundary Value Problem for Supercritical Flow of Viscoelastic Fluids of Maxwell Type

Michael Renardy, Ill-Posedness Resulting from Slip As a Possible Explanation of Melt Fracture

Michael Renardy, Compatibility Conditions at Corners Between Walls and Inflow Boundaries for Fluids of Maxwell Type

Rolf Rees, The Spectrum of Restricted Resolvable Designs with $r=2$

D. Lewis and J.C. Simo, Nonlinear stability of rotating pseudo-rigid bodies

Robert Hardt and David Kinderlehrer, Variational Principles with Linear Growth

San Yih Lin and Yisong Yang, Computation of Superconductivity in Thin Films

A. Narain, Pressure Driven Flow of Pure Vapor Undergoing Laminar Film Condensation Between Parallel Plates

P.J. Vassiliou, On Local Equivalence for Vector Field Systems 